\title{
Finite-Time Switching Control of Nonholonomic Mobile Robots for Moving Target Tracking Based on Polar Coordinates
}

\author{
Hua Chen $\left(\mathbb{D},{ }^{1}\right.$ Shen $X{ }^{2},{ }^{2}$ Lulu Chu, ${ }^{3}$ Fei Tong, ${ }^{3}$ and Lei Chen ${ }^{3}$ \\ ${ }^{1}$ Mathematics and Physics Department, Hohai University, Changzhou Campus, 213022, China \\ ${ }^{2}$ School of Mechanical and Electrical Engineering, Hohai University, Changzhou Campus, 213022, China \\ ${ }^{3}$ School of Internet of Things, Hohai University, Changzhou Campus, 213022, China \\ Correspondence should be addressed to Hua Chen; chenhua112@163.com
}

Received 3 April 2018; Revised 2 August 2018; Accepted 28 August 2018; Published 22 October 2018

Academic Editor: Daniela Paolotti

Copyright (c) 2018 Hua Chen et al. This is an open access article distributed under the Creative Commons Attribution License, which permits unrestricted use, distribution, and reproduction in any medium, provided the original work is properly cited.

In this paper, finite-time tracking problem of nonholonomic mobile robots for a moving target is considered. First of all, polar coordinates are used to characterize the distance and azimuth between the moving target and the robot. Then, based on the distance and azimuth transported from the sensor installed on the robot, a finite-time tracking control law is designed for the nonholonomic mobile robot by the switching control method. Rigorous proof shows that the tracking error converges to zero in a finite time. Numerical simulation demonstrates the effectiveness of the proposed control method.

\section{Introduction}

Addressing the problem of tracking a moving target is a challenging task which has attracted a continuously increasing attention in the control community [1]. Tracking of a moving target has its own complexities, mainly as follows: the movement of the target is more diverse and more dense and has large clutter density. The tracking of a ground moving target using nonholonomic robots has always been one of the forefront topics of great concern, because it has very important applications in cooperative robot reconnaissance [2], multirobot formation [3,4], and trajectory tracking [5-9]. Unmanned aerial robots (UARs) and unmanned ground robots (UGRs) [10-13] are often used to track a moving target. When UARs are in operation, they always rotate the track above the moving target, because the speed of UARs is much faster than that of the moving target. So there is a drawback of this tracking method. That is the lack of concealment. In contrast, the speed of UGRs is similar with that of the moving target and UGRs can maintain a certain distance from the moving target in the tracking process. In this case, concealment can also be guaranteed. Based on this superiority, UGRs are often prioritized when performing ground moving target tracking. The finite-time [14-16] tracking problem for nonholonomic mobile robots has been studied mostly in the contexts of optimality, controllability, and deadbeat control for several decades. Firstly, Tang et al. [17] presented BLF-Tan control laws for strict-feedback [18-21] nonlinear systems with output constraints. Based on the method of Tang, Wang et al. [12] proposed a generalized Tan-type barrier Lyapunov function to deal with the moving target tracking. However, most of the target tracking methods proposed in the existing papers can only guarantee that the error system asymptotically converges to zero, and the convergence speed often cannot meet the actual engineering requirements to achieve the expected tracking performance. This paper proposes a finite-time switching control tracking method to resolve this problem. In addition, the tracking objects are often nonholonomic robots in existing articles. The tracking objects studied in this paper are arbitrary moving targets. Compared with nonholonomic robots, tracking arbitrary moving targets is more research-oriented and more challenging because the tracking objects are no longer limited to nonholonomic robots, but can also be all moving objects such as people, and rolling balls, which has great research significance for practical engineering. 
In case the distance between the robot and the target is too small to prevent them from collision [12], this paper assumes that there is a minimum safety distance in that the distance between the robot and the target is always greater. In addition, due to the limited measurement range of the sensor, once the distance is greater than the maximum measurement of the sensor, it may lead to that the tracking error [22-27] is too large or may lead to tracking failure. In this article, a maximum distance is set to ensure that the distance between the robot and the target is always less. Similarly, the minimum azimuth and maximum azimuth angles between the robot and the target are set to meet that the azimuth is always between the minimum angle and the maximum angle. One of the novelties of this article is that the stricter constraints of the distance and the azimuth between the robot and the target are considered. Last appropriate control laws are set to meet that the distance and the azimuth between the robot and the moving target converge into the proper range in a finite time.

In this paper, a finite-time moving target tracking method is proposed by using the switching control method under polar coordinates without destroying the constraints of the system. The control law is set for the speed and angular velocity of nonholonomic mobile robots to ensure that the distance between the robot and the target and the azimuth converge to the ideal tracking distance and the ideal tracking azimuth in a finite time. The method has faster convergence rate and stronger robustness [28-32] to system uncertainty and external disturbance. The rigorous mathematic deduction and proof demonstrates the reliability of the method in this paper. Further simulation also further confirmed it. The main innovations and contributions of our research can be summarized as the following three points in this paper:

(1) A finite-time switching controller is set for the speed and angular velocity of the robot to track the mobile target in a finite time. Also, the tracking error can converge to zero in a finite time.

(2) Previous tracking objects are extended from nonholonomic robots to arbitrary moving targets in this article.

(3) The rigorous mathematical formula proves the reliability of the finite-time control law proposed in this paper. Further numerical simulation results also demonstrate the effectiveness of the control law.

This paper is organized as follows. Section 2 gives a formalization of the problem considered in this paper. Section 3 applies the stability results to the finite-time tracking control problem of nonholonomic mobile robots. Section 4 provides a numerical example and the corresponding simulation results of the proposed method, and finally, conclusions are given in Section 5.

\section{Problem Statement}

This paper studies the problem of tracking the moving targets with the nonholonomic mobile robots. The motion model of the nonholonomic mobile robots can be described by the following differential equations [3]:

$$
\begin{aligned}
& \dot{x}=v \cos \theta, \\
& \dot{y}=v \sin \theta, \\
& \dot{\theta}=w,
\end{aligned}
$$

where $[x, y]^{T} \in R^{2}$ is the Cartesian coordinate position of the nonholonomic mobile robots on the coordinate plane $\Psi$, and $\theta$ is its heading angle. $v$ and $w$ are the forward velocity and angular velocity of the nonholonomic mobile robots, respectively, from the horizontal axis.

The motion equation of the moving target can be described as [8]

$$
\begin{aligned}
& \dot{x}_{t}=v_{x}, \\
& \dot{y}_{t}=v_{y},
\end{aligned}
$$

where $\left[x_{t}, y_{t}\right]^{T} \in R^{2}$ is the position coordinate of the target on the coordinate plane $\Psi$, and $v_{x}$ and $v_{y}$ are the velocity components of the moving target along $X^{d}$ and $Y^{d}$ on the coordinate plane $\Psi$. In fact, both $v_{x}$ and $v_{y}$ are bounded variables.

Remark 1. If there are more than one robot, subscript $i$ should be used to number the robot; $v_{i}$ and $\theta_{i}$ represent the forward velocity and heading angle of the robot, respectively. For convenience, this article only studies the case of a single robot tracking a moving target.

Remark 2. This article assumes that the robot is equipped with an on-board sensor. It can measure the distance and the azimuth between the robot and the target.

Remark 3. According to Figure 1, we set the velocity vector of the initial state of the ground moving target in the third quadrant. The initial state speed of nonholonomic mobile robots is also in the third quadrant, and the robot is in the upper right direction with respect to the target. That is to say, the velocity vector of the robot is always in the third quadrant. Namely, $\theta$ is always in the range $(-\pi,-\pi / 2)$.

As shown in Figure 1, this article uses polar coordinates to describe the relative position between the robot and the target on the coordinate plane $\Psi$. The azimuth and distance of the robot and the target can be described as the following model.

$$
\begin{aligned}
& d=\sqrt{\left(x_{t}-x\right)^{2}+\left(y_{t}-y\right)^{2}}, \\
& \sigma=\arctan 2(Y, X),
\end{aligned}
$$

where $\arctan 2(x, y)$ is an inverse tangent function with four quadrants and its range is $(-\pi, \pi]$ by definition. Its 


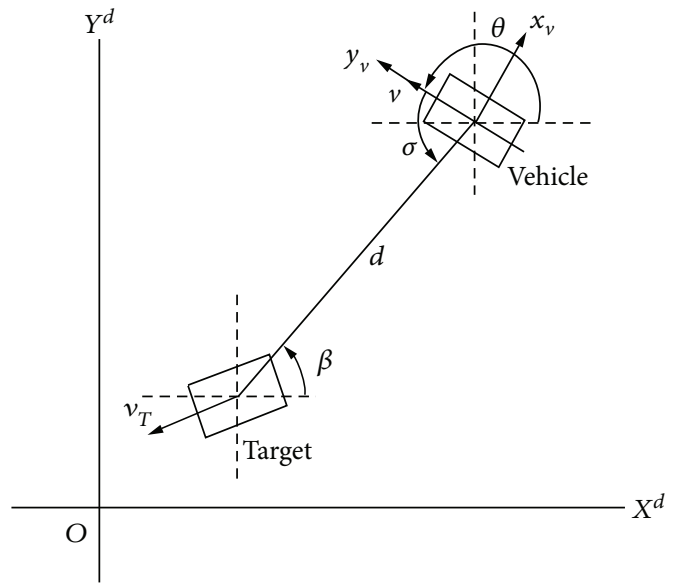

FIGURE 1: Tracking of the moving target.

accurate value is determined by the quadrant of the point $(x, y) . Y$ and $X$ satisfy the following equation:

$$
\left[\begin{array}{l}
X \\
Y
\end{array}\right]=\left[\begin{array}{cc}
\cos \theta & \sin \theta \\
-\sin \theta & \cos \theta
\end{array}\right]\left[\begin{array}{l}
x_{t}-x \\
y_{t}-y
\end{array}\right],
$$

$d$ and $\sigma$ are available according to the sensor installed on the robot. From Figure 1 , it can be obtained that $d, \sigma$, and $\beta$ satisfy the following equation:

$$
\sigma+\theta-\beta=\pi
$$

To facilitate the discussion of the main results, the following lemmas from existing literature are stated below.

Lemma 1. When $\eta=k_{1} \sin x+k_{2} \cos x$ is established, $\eta$ is always bounded. Where $k_{1}$ and $k_{2}$ are bounded variables, $x$ is any real number. (For complete, proof see the appendix).

Theorem 1. According to system (3), the derivative of $d$ and $\sigma$ with respect to time is

$$
\begin{aligned}
& \dot{d}=-v \cos \sigma-v_{x} \cos \beta-v_{y} \sin \beta, \\
& \dot{\sigma}=w \cos \theta+\frac{v}{d} \sin \sigma+\frac{1}{d}\left(v_{x} \sin \beta-v_{y} \cos \beta\right),
\end{aligned}
$$

Proof 1. For the convenience of calculation, substitutions need to be made.

$$
\begin{aligned}
& K_{d}=-v_{x} \cos \beta-v_{y} \sin \beta, \\
& K_{\sigma}=v_{x} \sin \beta-v_{y} \cos \beta,
\end{aligned}
$$

where $\beta$ is the angle between the line of the robot and the target with the direction of $X^{d}$. It has been mentioned above. $v_{x}$ and $v_{y}$ are bounded. Therefore, there are positive numbers $\omega_{1}$ and $\omega_{2}$ to satisfy the inequality.

$$
\left|v_{x}\right| \leq \omega_{1},\left|v_{y}\right| \leq \omega_{2},
$$

In combination with (8) and Lemma 1 , it can be obtained that there are positive numbers $\varsigma_{1}$ and $\varsigma_{2}$ that satisfy the inequalities below.

$$
\left|K_{d}\right| \leq \varsigma_{1},\left|K_{\sigma}\right| \leq \varsigma_{2}
$$

The above discussion can obtain that $K_{d}$ and $K_{\sigma}$ are also bounded.

(3) can be deformed into the following form after deformation.

$$
\begin{aligned}
d^{2} & =\left(x-x_{t}\right)^{2}+\left(y-y_{t}\right)^{2}, \\
\sigma & =\arctan 2(Y, X),
\end{aligned}
$$

According to the definition and (10), $\sigma$ satisfies the following equations.

$$
\begin{aligned}
& \cos \sigma=\frac{X}{\sqrt{X^{2}+Y^{2}}}, \\
& \sin \sigma=\frac{Y}{\sqrt{X^{2}+Y^{2}}} .
\end{aligned}
$$

Then, the derivatives of $d$ and $\sigma$ with respect to time are

$$
\begin{aligned}
& \dot{d}=\frac{\left(x-x_{t}\right)\left(v \cos \theta-v_{x}\right)+\left(y-y_{t}\right)\left(v \sin \theta-v_{y}\right)}{d}, \\
& \dot{\sigma}=\frac{X \dot{Y}-Y \dot{X}}{X^{2}+Y^{2}}=\frac{1}{d}(\dot{Y} \cos \sigma-\dot{X} \sin \sigma),
\end{aligned}
$$

After simplifying (4), it can be obtained that

$$
\begin{aligned}
X & =-\cos \theta\left(x-x_{t}\right)-\sin \theta\left(y-y_{t}\right), \\
Y & =\sin \theta\left(x-x_{t}\right)-\cos \theta\left(y-y_{t}\right), \\
x-x_{t} & =-X \cos \theta+Y \sin \theta, \\
y-y_{t} & =-X \sin \theta-Y \cos \theta .
\end{aligned}
$$

Then, the derivatives of $X$ with respect to time are

$$
\begin{aligned}
\dot{X}= & w \sin \theta\left(x-x_{t}\right)-\cos \theta\left(\dot{x}-\dot{x}_{t}\right) \\
& -w \cos \theta\left(y-y_{t}\right)-\sin \theta\left(\dot{y}-\dot{y}_{t}\right) .
\end{aligned}
$$

Substituting (1), (2), and (13) into (14), it can be obtained that

$$
\begin{aligned}
\dot{X}= & w \sin \theta(-X \cos \theta+Y \sin \theta)-\cos \theta\left(v \cos \theta-v_{x}\right) \\
& -w \cos \theta(-X \sin \theta-Y \cos \theta)-\sin \theta\left(v \sin \theta-v_{y}\right),
\end{aligned}
$$

(15) merged and simplified can be converted into

$$
\dot{X}=w Y-v_{x} \cos \theta+v_{y} \sin \theta .
$$


Similarly, it can be obtained that

$$
\dot{Y}=-w X-v_{x} \sin \theta+v_{y} \cos \theta
$$

Substituting (11), (13), (16), and (17) into (12), it can be obtained that

$$
\begin{aligned}
\dot{d}= & \frac{(Y \sin \theta-X \cos \theta)\left(v \cos \theta-v_{x}\right)}{\sqrt{X^{2}+Y^{2}}} \\
& -\frac{(X \sin \theta+Y \cos \theta)\left(v \sin \theta-v_{y}\right)}{\sqrt{X^{2}+Y^{2}}} \\
= & (\sin \sigma \sin \theta-\cos \sigma \cos \theta)\left(v \cos \theta-v_{x}\right) \\
& -(\cos \sigma \sin \theta+\sin \sigma \cos \theta)\left(v \sin \theta-v_{y}\right) \\
= & -\cos (\sigma+\theta)\left(v \cos \theta-v_{x}\right)-\sin (\sigma+\theta)\left(v \sin \theta-v_{y}\right), \\
\dot{\sigma}= & \frac{1}{d}(\dot{Y} \cos \sigma-\dot{X} \sin \sigma) \\
= & \frac{1}{d}\left[\cos \sigma\left(-w X-v_{x} \sin \theta+v_{y} \cos \theta\right)\right. \\
& \left.-\sin \sigma\left(w Y+v_{x} \cos \theta+v_{y} \sin \theta-v\right)\right] \\
= & -w \cos (\sigma-\beta)-\frac{v_{x}}{d} \sin (\sigma+\theta) \\
& +\frac{v_{y}}{d} \cos (\sigma+\theta)-\frac{v \sin \sigma}{d}
\end{aligned}
$$

Substituting (5) into (18), after some trigonometric transformations, it can be obtained that

$$
\begin{aligned}
& \dot{d}=-v \cos \sigma+K_{d}, \\
& \dot{\sigma}=w \cos \theta+\frac{v}{d} \sin \sigma+\frac{1}{d} K_{\sigma} .
\end{aligned}
$$

The above is a complete proof of Theorem1.

By definition, the range of $d$ is $(0,+\infty)$, and the range of $\sigma$ is $(-\pi, \pi]$. But in fact when $d$ is too small, the robot will collide with the target which means tracking will not continue. So there is a minimum safety distance $d_{\min }$ and $d$ is always greater than it. In addition, due to the limitation of the measurement range of the on-board sensor, there is a maximum measurement distance $d_{\max }$ keeping $d$ always smaller. During the tracking process, the system always keeps $d$ in the range $\left(d_{\min }, d_{\max }\right)$. Similarly, the measurement range of the sensor is bounded. So $\sigma$ is also a bounded variable. There is a minimum azimuth $\sigma_{\min }$ and a maximum azimuth $\sigma_{\max }$ satisfying that $\sigma$ is always between them in the progress of tracking.

\section{Main Results}

This section presents a novel method solving the problem of ground moving target tracking with constraints on $d$ and $\sigma$ using nonholonomic mobile robots. This paper uses the method of switching control to set the control law so that the convergence error converges to zero in a finite time. The convergence error includes distance error and azimuth error.
Lemma 2 [32-34]. Consider a first-order system $\dot{\tau}=\psi$ where $\tau$ is a positive value function of time. Moreover, there is a positive number $\psi_{M}$ satisfying $|\psi| \leq \psi_{M}$. For a certain number $\tau(0)$, there is always a positive number $D_{0}$ satisfying the inequality $|\tau(0)| \leq D_{0}$. Set the continuous and saturated control law $\psi=-\kappa \operatorname{sgn}(\tau)|\tau|^{\varsigma}$ where $\kappa$ and $\zeta$ are design parameters and satisfy $\kappa \leq\left(\psi_{M} / D_{0}^{\zeta}\right), 0<\zeta<1$. There is a finite time $T_{0}$ satisfying $T_{0}=|\tau(0)|^{1-\zeta} / \kappa(1-\zeta) \leq D_{0}{ }^{1-\zeta} / \kappa(1-\zeta)$, such that

$$
\lim _{t \rightarrow T_{0}} \tau=0, \tau \equiv 0\left(t \geq t_{0^{-}}\right)
$$

3.1. Switching Controller DesignStep 1. According to Remark 3 , it can be obtained that $\theta \neq \pi / 2$ and $\theta \neq-\pi / 2$ in the process of tracking. Moreover, $\cos \theta \neq 0$ can be obtained. Then, consider a replacement $w \cos \theta=\bar{w}$. Take the following control law on $v$ and $\bar{w}$.

$$
\begin{aligned}
v & =0 \\
\bar{w} & =-\frac{K_{\sigma}}{d}-\kappa_{1} \operatorname{sgn}(\sigma)|\sigma|^{\zeta_{1}},
\end{aligned}
$$

where $\kappa_{1} \leq \sigma^{\max } / D^{\zeta_{1}}, 0 \leq \zeta_{1} \leq 1$. When $\sigma$ converges to zero, go to Step 2.

Step 2. $\sigma$ converges to zero and $\dot{d}=-v+K_{d}$. Then, take the following control law.

$$
\begin{gathered}
v=K_{d}+\kappa_{2} \operatorname{sgn}(d)|d|^{\zeta_{2},} \\
\bar{w}=-\frac{K_{\sigma}}{d}-\kappa_{1} \operatorname{sgn}(\sigma)|\sigma|^{\zeta_{1}},
\end{gathered}
$$

where $\kappa_{2} \leq d^{\max } / D^{\zeta_{2}}, 0 \leq \zeta_{2} \leq 1$

Case 1. If $\left|d-d_{\rho}\right|<\varepsilon_{1}$, where $\varepsilon_{1}$ is an arbitrary positive number, then go to Step 3 .

Case 2. If there is a positive number $\varepsilon_{2}$ satisfying $\left|d-d_{\rho}\right|>\varepsilon_{1}$, then go to Step 2 again.

Step 3. Take the following control law.

$$
\begin{aligned}
v & \equiv K_{d}, \\
\bar{w} & =-\frac{K_{\sigma}}{d}-\kappa_{1} \operatorname{sgn}(\sigma)|\sigma|^{\zeta_{1}} .
\end{aligned}
$$

Theorem 2. The system (6) can be transformed to the following system by setting the switching control law.

$$
\begin{aligned}
& \dot{d}=-\kappa_{1} \operatorname{sgn}(d)|d|^{\zeta_{1}}, \\
& \dot{\sigma}=-\kappa_{2} \operatorname{sgn}(\sigma)|\sigma|^{\zeta_{2}} .
\end{aligned}
$$

According to Lemma 2, rigorous mathematical proof shows that the tracking error converges to zero in a limited 
time. It can be obtained that $\exists t_{1}>0$ when $t>t_{1}, d$ converges to ideal tracking distance $d_{\rho}$ and $\sigma$ converges to zero.

Proof 2. Substitute (21) into (19). It can be obtained that $\dot{\sigma}=-\kappa_{1} \operatorname{sgn}(\sigma)|\sigma|^{\zeta_{1}}$. Consider the Lyapunov stability decision function $V=1 / 2 \sigma^{2}$. Because $\sigma$ is a function of time, $V$ is also a function of time. Then, the derivative of $V$ with respect to time is

$$
\begin{aligned}
\dot{V} & =\sigma \dot{\sigma}=-\kappa_{1} \sigma \operatorname{sgn}(\sigma)|\sigma|^{\zeta_{1}}=-\kappa_{1}|\sigma|^{\zeta_{1}+1} \\
& =-\kappa_{1}\left(\sigma^{2}\right)^{\zeta_{1}+1 / 2}=-\kappa_{1}(2 V)^{\zeta_{1}+1 / 2} .
\end{aligned}
$$

According to (25), it can be obtained that $V$ is a decreasing function of time. Then, (25) can be transformed into

$$
\frac{d V}{(V)^{\zeta_{1}+1 / 2}}=-2^{\zeta_{1}+1 / 2} \kappa_{1} d t
$$

Integrate both sides of (26); it can be obtained that

$$
\begin{aligned}
\int_{V(0)}^{V} V^{-1+\zeta_{1} / 2} d V & =\int_{0}^{t}-2^{\zeta_{1}+1 / 2} \kappa_{1} d t \frac{1}{1-\left(1+\zeta_{1} / 2\right)} V^{1-\zeta_{1} / 2} \\
& =-2^{\zeta_{1}+1 / 2} \kappa_{1} t+\frac{1}{1-\left(1+\zeta_{1} / 2\right)}(V(0))^{1-\zeta_{1} / 2}
\end{aligned}
$$

After some calculations, (27) can be simplified as

$$
V^{1-\zeta_{1} / 2}=-2^{\zeta_{1}-1 / 2} \kappa_{1}(1-\zeta) t+(V(0))^{1-\zeta_{1} / 2}
$$

It can be seen from (28) that $V$ converges to zero when $V$ converges to zero as $t$ tends to $T_{1}$. According to Lemma 1 , it can be obtained that $T_{1}$ is a finite value. The above proof shows that $V$ converges to zero in a finite time. According to Lyapunov stability decision function $V=1 / 2 \sigma^{2}, \sigma$ also converges to zero in a finite time.

In Step 2, substitute (22) into (19). It can be obtained that $\dot{d}=-\kappa_{2} \operatorname{sgn}(d)|d|^{\zeta_{2}}$. Similar to the convergence of $\sigma, d$ will decrease over time until it converges to $d_{\rho}$ in a finite time. Depending on the data returned by the sensors mounted on the robot, it can be obtained whether $d$ has reached the proper tracking distance. The setting of Case 1 and Case 2 can ensure tracking accuracy.

After the above repeated switching control, $d$ will always converge to $d_{\rho}$ in a finite time. When $d$ converges to $d_{\rho}$, go to Step 3.

After Step 1, $\sigma$ has converged to zero in a finite time. So $\sigma \equiv 0$ is always satisfied in the control laws of Step 2 and Step 3. By the control law of Step 3, the distance between the robot and the target will always be at the ideal tracking distance.

The above rigorous proof fully proves Theorem 2 .

In order to better understand the idea of the controller, this paper designed a flow chart (Figure 2).

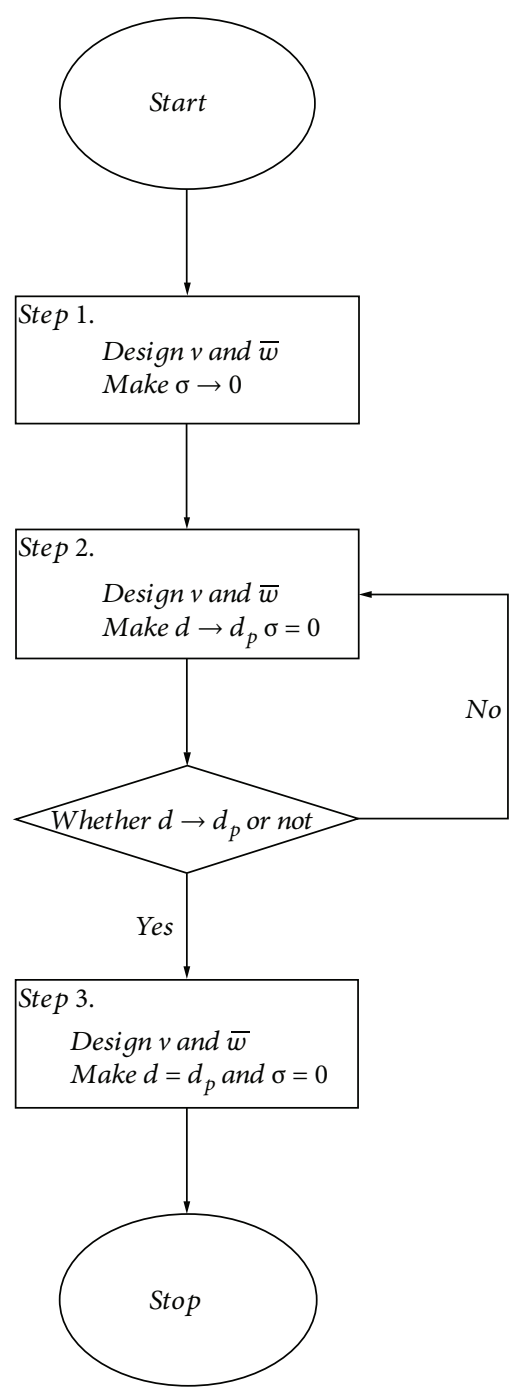

Figure 2

Remark 4. Note that in Theorem 2 and Lemma 2, the gains $\zeta_{1}, \zeta_{2}, \kappa_{1}$ and $\kappa_{2}$ are not explicitly given. Practically, we can choose the appropriate gains by a simulation-tested method, as suggested in the work of Levant [35], for example, a group of available parameters $\varsigma_{1}=0.5, \varsigma_{1}=0.25, \kappa_{1}=1, \kappa_{2}=2$.

For your convenience review, we make a table below to explain the source for each parameter selection (Table 1).

\section{Simulations}

In this section, we consider the tracking error $\sigma$ and $d^{*}=d-d_{\rho}$ based on the proposed finite-time tracking controller for system (1) to ensure that $d$ in system (6) converges to $d_{\rho}$ and $\sigma$ converges to zero. We demonstrate the effectiveness of our method in tracking moving targets by MATLAB simulation.

The simulation is carried out in which a robot is randomly distributed in the coordinate plane $\Psi$ at first and finally should achieve the required cooperative circumnavigation stated in Sections 2 and 3. 
TABLE 1

\begin{tabular}{lc}
\hline Parameters & Source of each parameter \\
\hline$\omega_{1}>0, \omega_{2}>0$ & From (8) \\
$\kappa_{1}>0, \kappa_{2}>0$ & From Lemma 2 and Theorem 2 \\
$\zeta_{1} \in(0,1), \zeta_{2} \in(0,1)$ & From Lemma 2 and Theorem 2 \\
\hline
\end{tabular}

In the following simulation, we assume that the ideal tracking distance is chosen to be given by $d_{\rho}=0.2$. The initial position of the robot is $[0,0]^{T}$ and the initial position of the target is $[0,10]^{T}$. We select design parameters $\kappa_{1}=1, \kappa_{2}=2$, $\varsigma_{1}=0.5$ and $\varsigma_{2}=0.25$. The speed of the target is time-varying:

$$
\begin{aligned}
& \dot{x}_{t}=0.5+0.25 \cos (0.5 t), \\
& \dot{y}_{t}=0.4\left(1.2-\tanh (0.2 t-3)^{2}\right) .
\end{aligned}
$$

Under the switching control law (21) and (22), the simulation result shows that Figure 3 shows that the tracking azimuth $\sigma$ can converge to zero in a finite time $t \leq 8 s$ in Step 1 and is kept in Step 2 until it is driven to zero in the last step. In Figure 4, it shows that tracking distance $d$ is stabilized to a constant $\left(d=d_{\rho}=0.2\right)$ in a finite time $t \leq 15 \mathrm{~s}$. Moreover, Figure 5 also shows that tracking error system $d^{*}$ converges to the neighborhood of zero in a finite time in which the converging speed is very fast. Figure 6 shows the complete simulation result of the entire switching controller. Finally, Figure 7 shows the trajectories of the robot and target in the plane in which it can be obtained that the tracking task can be achieved in a finite time.

In order to reflect the superiority of the method proposed in this paper, we compare the controller of this paper with the control law in [12], and Figure 8 shows that the error system converges to zero when $t \geq 20 \mathrm{~s}$ under the control law in [35]. Combining Figures 1 and 3, we can clearly see that the proposed switch control law has a faster error convergence rate than [12].

In actual engineering, the robot is often disturbed by noise. In Figures 9, 10, and 11, we consider the situation with random noise disturbance. The simulation results show that the control method proposed in this paper can still complete the task of tracking for the moving target in a short time in Figure 8. Figures 9 and 10 show that the error system can converge to zero in a very short time under the control laws proposed in this paper.

The numerical simulation results show that not only can the controller proposed in this paper make the error system converge to zero in a very short time but also the system has better anti-interference under this controller.

\section{Conclusion}

This paper studies the problem of moving target tracking using nonholonomic ground robots. Based on the polar coordinates, the switching control method is used to design a reasonable control law which can achieve that the error of distance and azimuth between the robot and the target converges to zero in a finite time. Compared with the

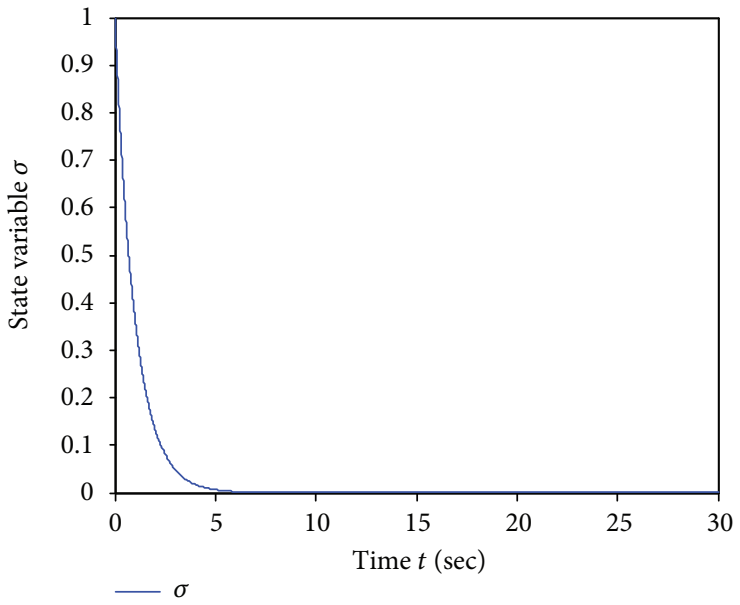

FIgURE 3: The response of the tracking variable $\sigma$ with respect to time.

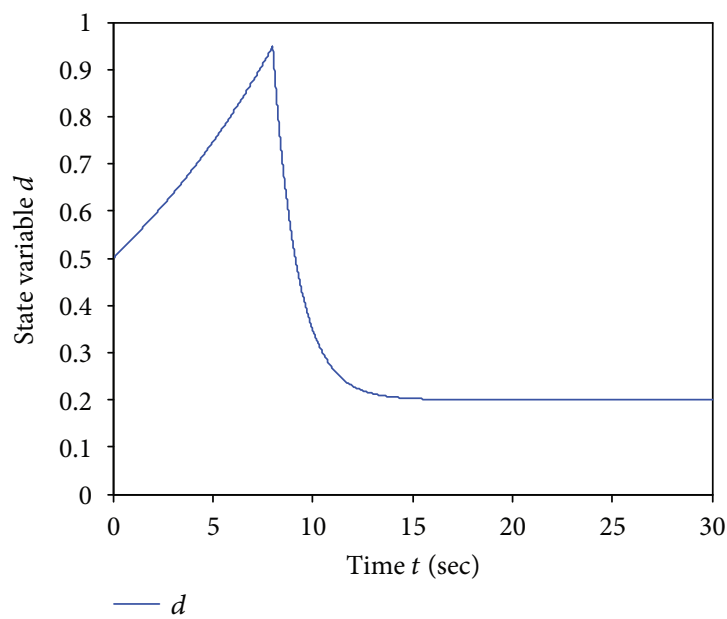

FIgURE 4: The convergence of tracking distance $d$ with respect to time.

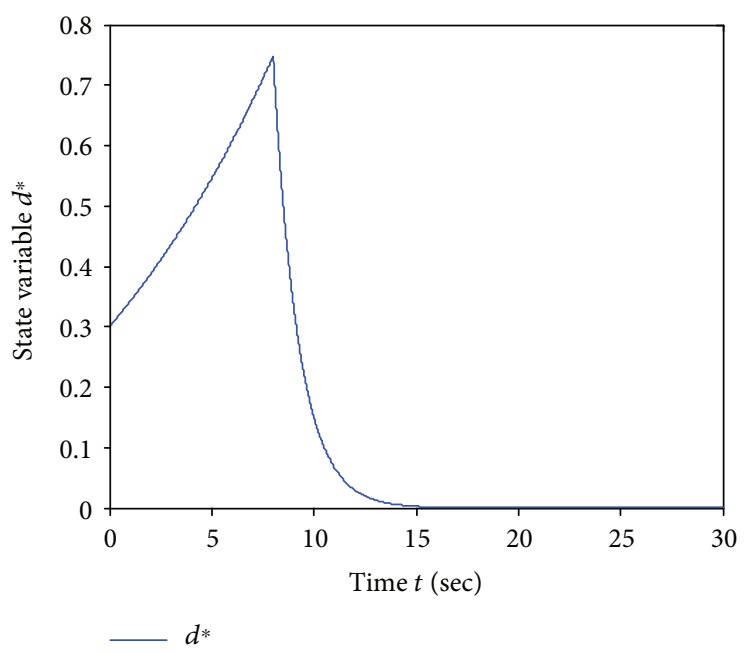

Figure 5: The stability of distance error $d^{*}$ with respect to time. 


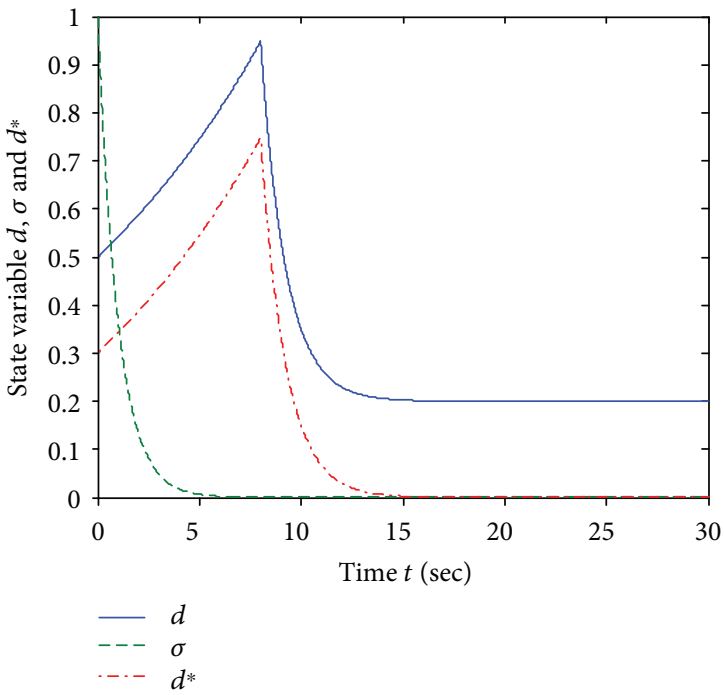

Figure 6: The complete response of tracking variables $\left(d, \sigma, d^{*}\right)$ with respect to time.

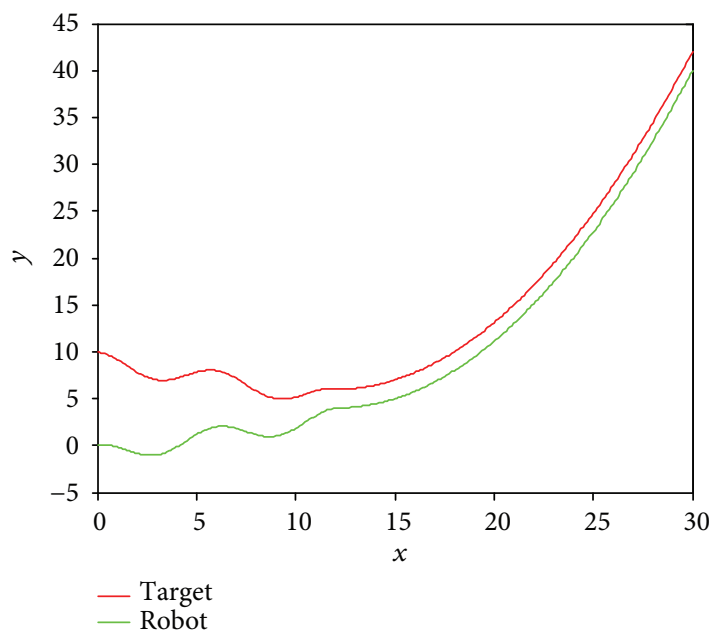

FIGURE 7: The trajectories of robot and target in the plane.

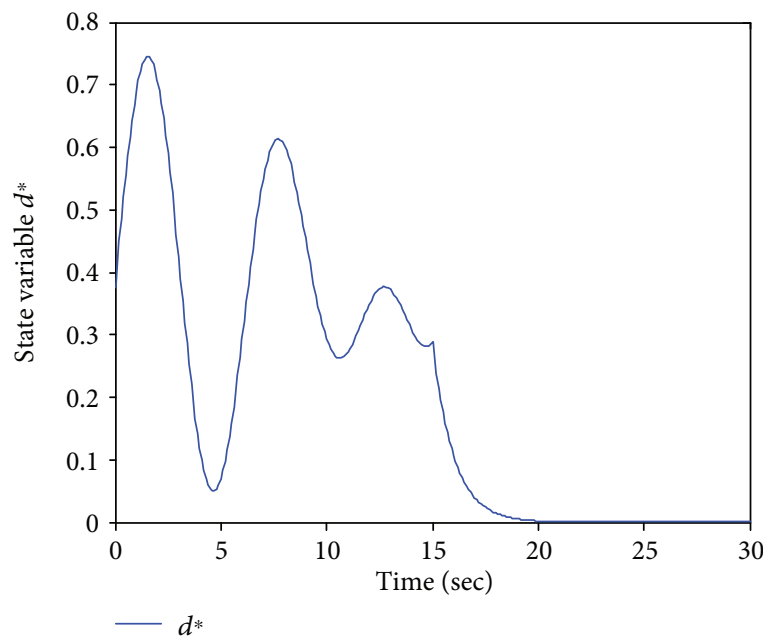

FIGURE 8: The stability of distance error $d^{*}$ with respect to time in.

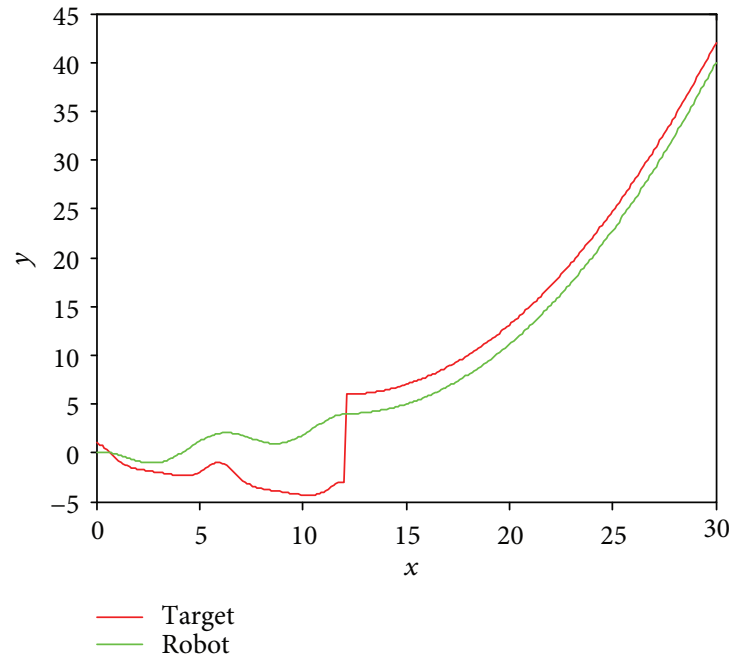

FIgURE 9: The trajectories of robot and target with random noise disturbance.

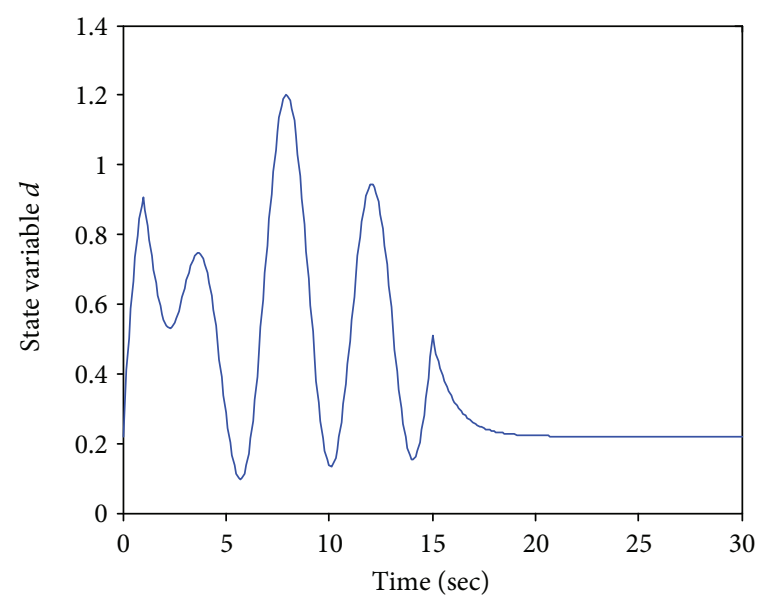

$-d$

FIgURE 10: The distance of robot and target with random noise disturbance.

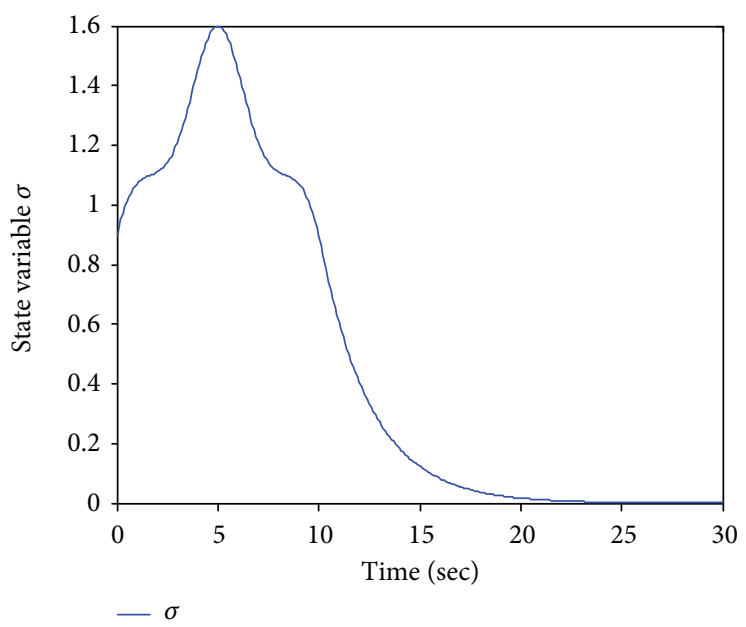

FIgURE 11: The azimuth of robot and target with random noise disturbance. 
traditional tracking algorithm, the proposed method in this article not only guarantees that the tracking error can converge to the neighborhood of zero in a finite time but also extends the previous tracking object from a nonholonomic robot to an arbitrary moving target. This paper also considers that the distance and azimuth between the robot and the target is limited, which is very practical for realistic engineering. The collision problem between robots has not been considered in this article and will be the focus of attention in future research.

\section{Appendix}

The complete proof of Theorem 1:

Proof 3. Because $k_{1}$ and $k_{2}$ are bounded variables, it may be assumed that there are positive numbers $\lambda_{1}$ and $\lambda_{2}$ satisfying the following inequality

$$
\begin{aligned}
& \left|k_{1}\right| \leq \lambda_{1} \\
& \left|k_{2}\right| \leq \lambda_{2}
\end{aligned}
$$

Hence,

$$
\begin{aligned}
|\eta| & =\left|k_{1} \sin x+k_{2} \cos x\right| \leq\left|k_{1} \sin x\right|+\left|k_{2} \cos x\right| \\
& \leq\left|k_{1}\right|+\left|k_{2}\right| \leq \lambda_{1}+\lambda_{2},
\end{aligned}
$$

The above proof shows that $\eta$ is bounded and Theorem 1 is true.

\section{Data Availability}

The data used to support the findings of this study are available from the corresponding author upon request.

\section{Conflicts of Interest}

The authors declare that they have no conflicts of interest.

\section{Acknowledgments}

This work was supported by the National Natural Science Foundation of China $(61304004,61503205)$, the Changzhou Sci\&Tech Program (CJ20160013), the Fundamental Research Funds for the Central Universities (2017B15114), and the Changzhou Key Laboratory of Aerial Work Equipment and Intellectual Technology (CLAI201803).

\section{References}

[1] J. Kim and Y. Kim, "Moving ground target tracking in dense obstacle areas using UAVs," IFAC Proceedings Volumes, vol. 41, no. 2, pp. 8552-8557, 2008.

[2] T. Balch and R. C. Arkin, "Behavior-based formation control for multirobot teams," IEEE Transactions on Robotics and Automation, vol. 14, no. 6, pp. 926-939, 1998.

[3] D. Zhao and T. Zou, "A finite-time approach to formation control of multiple mobile robots with terminal sliding mode,"
International Journal of Systems Science, vol. 43, no. 11, pp. 1998-2014, 2012.

[4] Y. Hong and Z.-P. Jiang, "Finite-time stabilization of nonlinear systems with parametric and dynamic uncertainties," IEEE Transactions on Automatic Control, vol. 51, no. 12, pp. 19501956, 2006.

[5] T. Fukao, H. Nakagawa, and N. Adachi, "Adaptive tracking control of a nonholonomic mobile robot," IEEE Transactions on Robotics and Automation, vol. 16, no. 5, pp. 609-615, 2000.

[6] B. S. Park, S. J. Yoo, J. B. Park, and Y. H. Choi, "A simple adaptive control approach for trajectory tracking of electrically driven nonholonomic mobile robots," IEEE Transactions on Control Systems Technology, vol. 18, no. 5, pp. 1199-1206, 2010.

[7] H. Chen, S. Ding, X. Chen, L. Wang, C. Zhu, and W. Chen, "Global finite-time stabilization for nonholonomic mobile robots based on visual servoing," International Journal of Advanced Robotic Systems, vol. 11, no. 11, p. 180, 2014.

[8] J. Li and C. Qian, "Global finite-time stabilization by dynamic output feedback for a class of continuous nonlinear systems," IEEE Transactions on Automatic Control, vol. 51, no. 5, pp. 879-884, 2006,".

[9] S. Ding, L. Liu, and W. X. Zheng, "Sliding mode direct yaw-moment control design for in-wheel electric vehicles," IEEE Transactions on Industrial Electronics, vol. 64, no. 8, pp. 6752-6762, 2017.

[10] P. Doherty and P. Rudol, "A UAV search and rescue scenario with human body detection and geolocalization," in AI 2007: Advances in Artificial Intelligence. AI 2007. Lecture Notes in Computer Science, vol 4830, M. A. Orgun and J. Thornton, Eds., Springer, Berlin, Heidelberg, 2007.

[11] S.-O. Lee, Y.-J. Cho, M. Hwang-Bo, B.-J. You, and S.-R. Oh, "A stable target-tracking control for unicycle mobile robots," in Proceedings. 2000 IEEE/RSJ International Conference on Intelligent Robots and Systems (IROS 2000) (Cat. No.00CH37113), pp. 1822-1827, Takamatsu, Japan, October-November 2000.

[12] Y. Wang, D. Wang, and B. C. Ng, "Finite time moving target tracking using nonholonomic vehicles with distance and bearing angle constraints," in 2017 American Control Conference (ACC), Seattle, WA, USA, May 2017.

[13] V. Gazi and R. Ordónez, "Target tracking using artificial potentials and sliding mode control," International Journal of Control, vol. 80, no. 10, pp. 1626-1635, 2007.

[14] O. Mofid and S. Mobayen, "Adaptive sliding mode control for finite-time stability of quad-rotor UAVs with parametric uncertainties," ISA Transactions, vol. 72, pp. 1-14, 2018.

[15] F. Bayat, S. Mobayen, and S. Javadi, "Finite-time tracking control of nth-order chained-form non-holonomic systems in the presence of disturbances," ISA Transactions, vol. 63, pp. 78-83, 2016.

[16] S. Mobayen and S. Javadi, "Disturbance observer and finitetime tracker design of disturbed third-order nonholonomic systems using terminal sliding mode," Journal of Vibration and Control, vol. 23, no. 2, pp. 181-189, 2017.

[17] Z.-L. Tang, K. P. Tee, and W. He, “Tangent barrier lyapunov functions for the control of output-constrained nonlinear systems," IFAC Proceedings Volumes, vol. 46, no. 20, pp. 449-455, 2013.

[18] R. M. Murray and S. S. Sastry, "Nonholonomic motion planning: steering using sinusoids," IEEE Transactions on Automatic Control, vol. 38, no. 5, pp. 700-716, 1993. 
[19] Y. P. Tian and S. Li, "Exponential stabilization of nonholonomic dynamic systems by smooth time-varying control," Automatica, vol. 38, no. 7, pp. 1139-1146, 2002.

[20] A. Teel, R. Murry, and G. Walsh, "Nonholonomic control systems: from steering to stabilization with sinusoids," in [1992] Proceedings of the 31st IEEE Conference on Decision and Control, pp. 1603-1609, Tucson, AZ, USA, December 1992.

[21] O. J. Sordalen and O. Egeland, "Exponential stabilization of nonholonomic chained systems," IEEE Transactions on Automatic Control, vol. 40, no. 1, pp. 35-49, 1995.

[22] Z. Qu and J. Dorsey, "Robust tracking control of robots by a linear feedback law," IEEE Transactions on Automatic Control, vol. 36, no. 9, pp. 1081-1084, 1991.

[23] Y. Wu, B. Wang, and G. D. Zong, "Finite-time tracking controller design for nonholonomic systems with extended chained form," IEEE Transactions on Circuits and Systems II: Express Briefs, vol. 52, no. 11, pp. 798-802, 2005.

[24] M. Ou, H. Du, and S. Li, "Finite-time formation control of multiple nonholonomic mobile robots," International Journal of Robust and Nonlinear Control, vol. 24, no. 1, pp. 140-165, 2014.

[25] D. Chwa, "Sliding-mode tracking control of nonholonomic wheeled mobile robots in polar coordinates," IEEE Transactions on Control Systems Technology, vol. 12, no. 4, pp. 637644, 2004.

[26] S. Mobayen, "Finite-time tracking control of chained-form nonholonomic systems with external disturbances based on recursive terminal sliding mode method," Nonlinear Dynamics, vol. 80, no. 1-2, pp. 669-683, 2015.

[27] S. Ding, W. X. Zheng, J. Sun, and J. Wang, "Second-order sliding-mode controller design and its implementation for Buck converters," IEEE Transactions on Industrial Informatics, vol. 14, no. 5, pp. 1990-2000, 2018.

[28] Y. Hong, "Finite-time stabilization and stabilizability of a class of controllable systems," Systems \& Control Letters, vol. 46, no. 4, pp. 231-236, 2002.

[29] S. P. Bhat and D. S. Bernstein, "Finite-time stability of continuous autonomous systems," SIAM Journal on Control and Optimization, vol. 38, no. 3, pp. 751-766, 2000.

[30] S. P. Bhat and D. S. Bernstein, "Continuous finite-time stabilization of the translational and rotational double integrators," IEEE Transactions on Automatic Control, vol. 43, no. 5, pp. 678-682, 1998.

[31] H. Chen, C. Wang, Z. Liang, D. Zhang, and H. Zhang, "Robust practical stabilization of nonholonomic mobile robots based on visual servoing feedback with inputs saturation," Asian Journal of Control, vol. 16, no. 3, pp. 692-702, 2014.

[32] S. Ding and S. Li, "Second-order sliding mode controller design subject to mismatched term," Automatica, vol. 77, pp. 388-392, 2017.

[33] Z. H. O. N. G.-P. I. N. G. JIANGdagger and H. Nijmeijer, "Tracking control of mobile robots: a case study in backstepping," Automatica, vol. 33, no. 7, pp. 1393-1399, 1997.

[34] Y. Hong, J. Huang, and Y. Xu, "On an output feedback finitetime stabilization problem," IEEE Transactions on Automatic Control, vol. 46, no. 2, pp. 305-309, 2001.

[35] A. Levant, "Higher-order sliding modes, differentiation and output-feedback control," International Journal of Control, vol. 76, no. 9-10, pp. 924-941, 2003. 


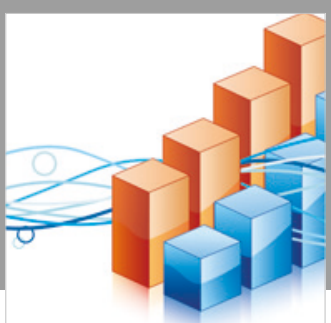

Advances in

Operations Research

\section{-n-m}
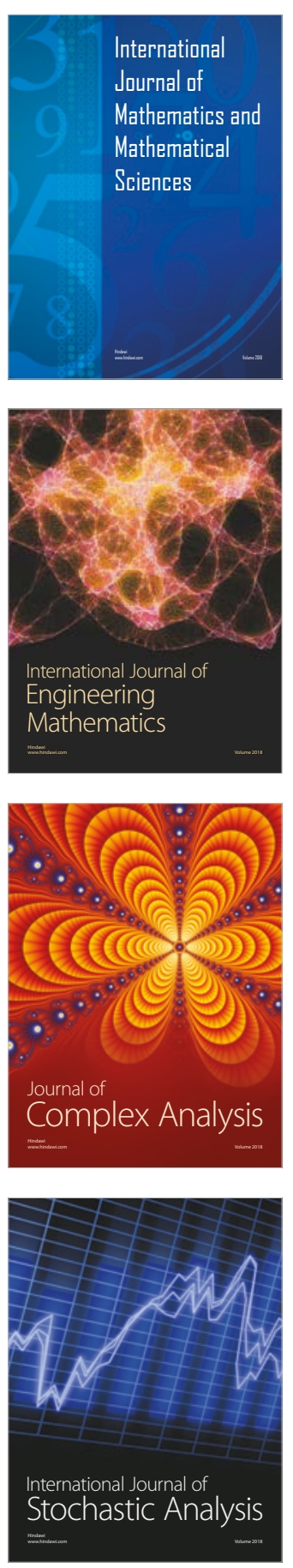
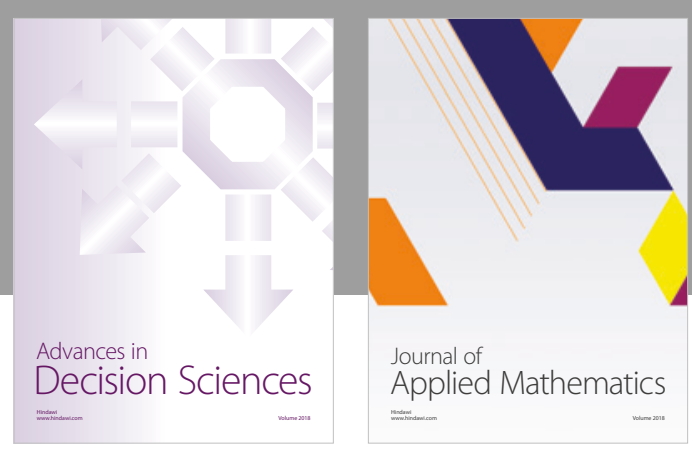

Journal of

Applied Mathematics
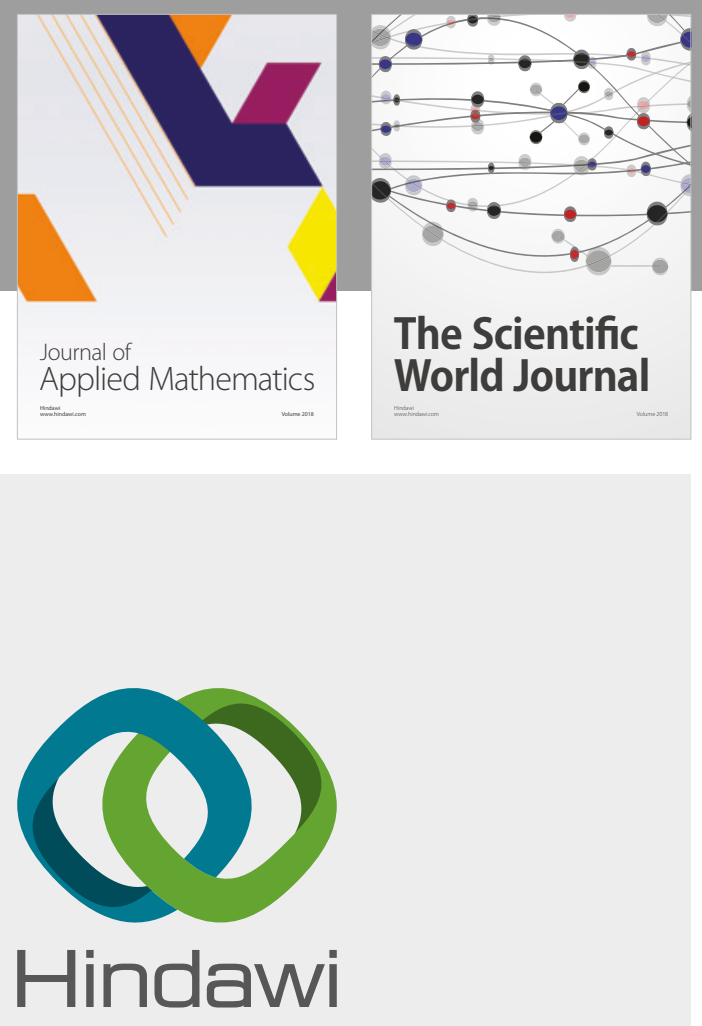

Submit your manuscripts at

www.hindawi.com

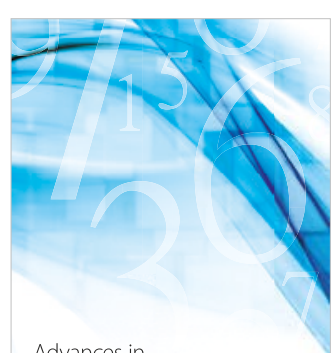

Advances in
Numerical Analysis
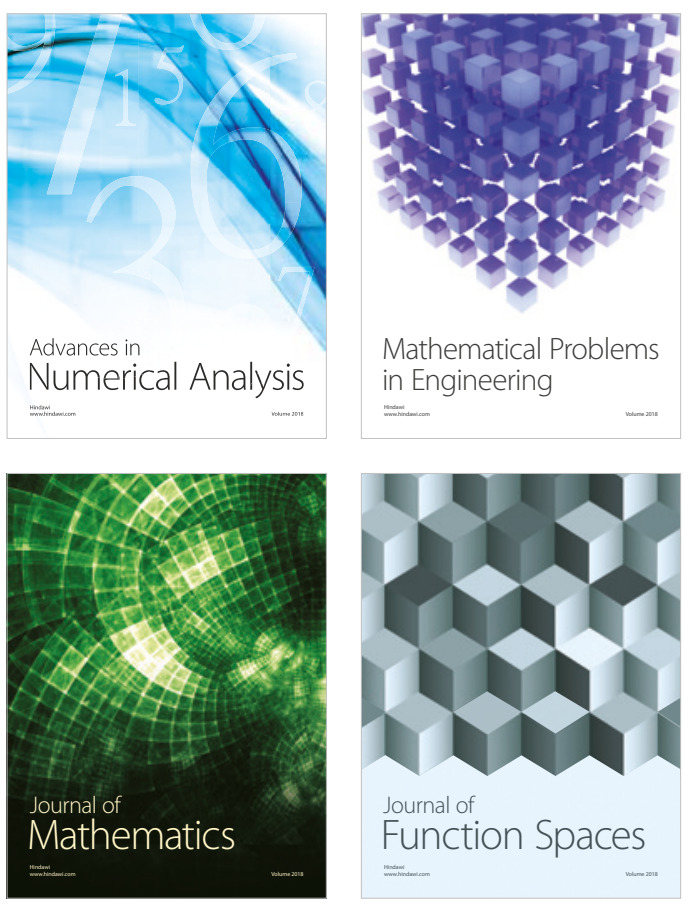

Mathematical Problems in Engineering

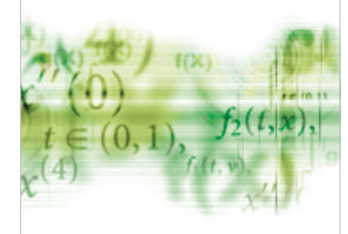

International Journal of

Differential Equations

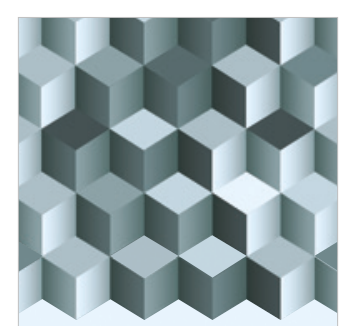

Journal of

Function Spaces
The Scientific

World Journal

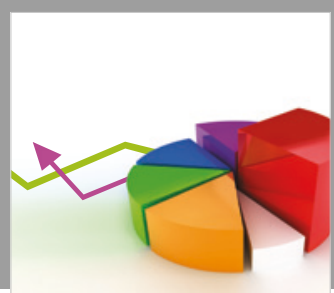

Journal of

Probability and Statistics
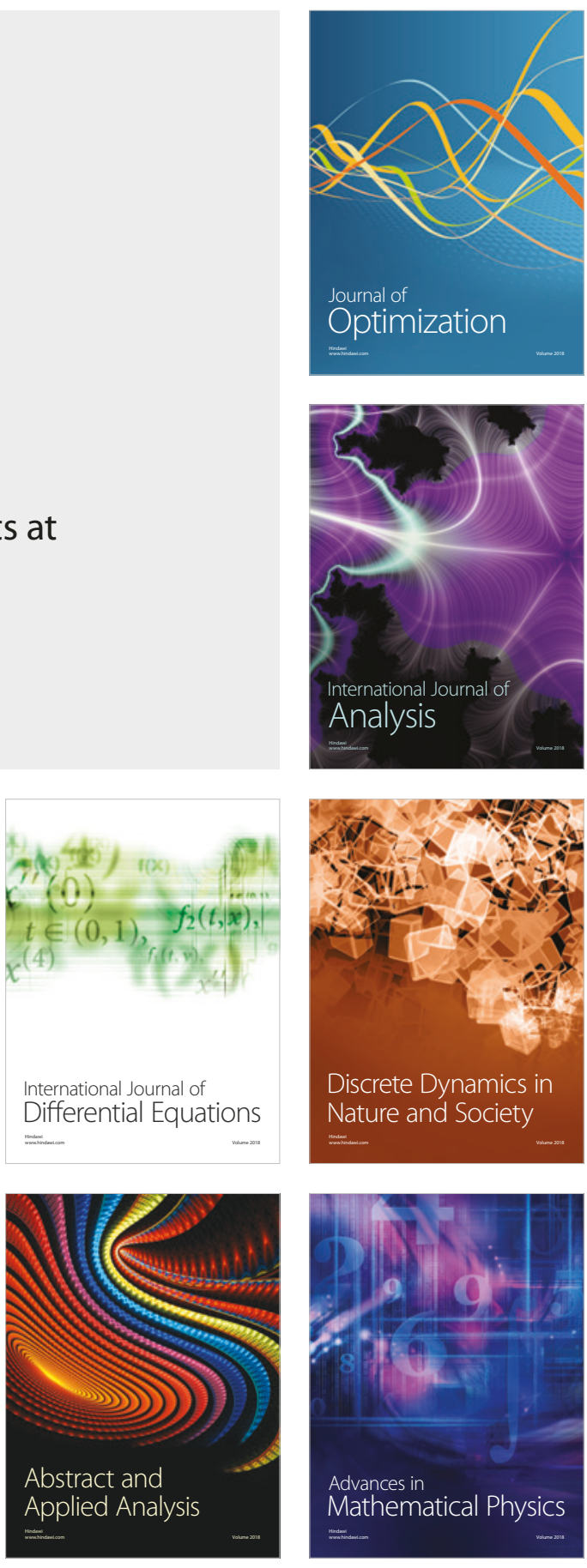